Enabling Context for Chinese Paintings

\title{
DESIGN PROBLEM
}

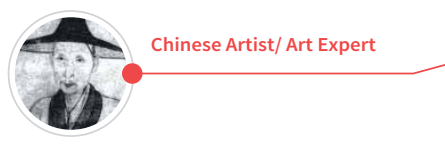

Bada Shanren

prominent traditional Chinese painter of the Ming dynasty

“ The essence of art should not be judged by likeliness to an object, but by the emotions and intelligence the art embodies.

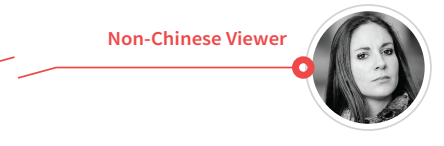

museum visitor 1

Chinese art section, University of Michigan Musuem of Art

॥ It is too different, too far away from me. I need something more personal.

There is currently a disconnect between Chinese artists and experts and non-Chinese viewers of traditional Chinese paintings, largely due to the poor communication of the context necessary to facilitate aesthetic appreciation of this unique art form.

\section{OUR APPROACH}

Our design, titled "Rice Paper", helps bridge the cultural disconnect between traditional Chinese painting artists or art experts and non-Chinese viewers.

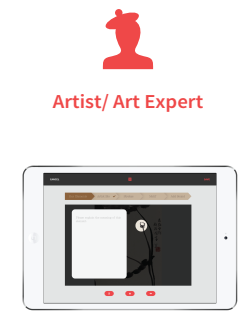

Use the application to create a booklet highlighting key elements, artist bio, strokes, and motif

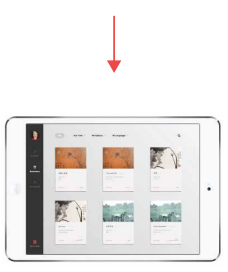

Make an electronic mockup of the booklet available in the in-app bookstore

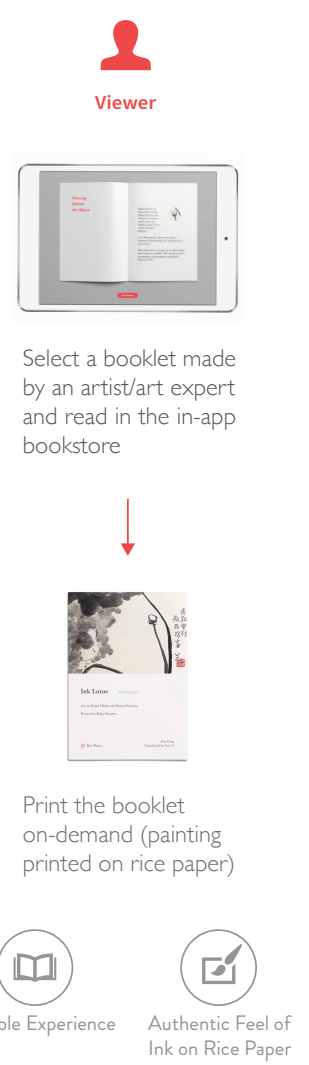

Rice Paper complements a painting, attaching context that is otherwise lost. It helps Chinese painting artists and experts to express the values and meanings to a wider audience and enables non-Chinese viewers to explore the essence of the art and the culture with a tangible experience.
Hariharan Subramonyam

Yuncheng Shen

Samantha Lauren Jones

University of Michigan

School of Information

\section{RESEARCH}

\section{- Participatory Design with Chinese Painter}

We held a participatory design session with the director of the Wuhan Art Museum and prominent Chinese painter, Fan Feng. Our goal was to observe the approach that a Chinese artist would take and the elements that they would highlight when explaining a painting.

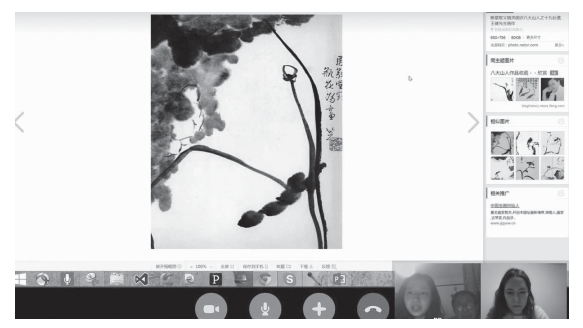

- Participatory Design with Cultural Educator

We also worked with Carol Stepanchuk, the Outreach Coordinator at the University of Michigan Center for Chinese Studies, to understand how Chinese paintings are taught in a non-Chinese context.

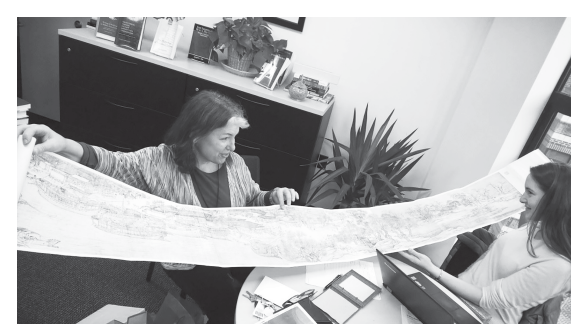

\section{- Interviews with Museum Visitors}

We interviewed visitors to the Chinese painting exhibits at Univerisity of Michigan Museum of Art and The Chicago Institute of Art.

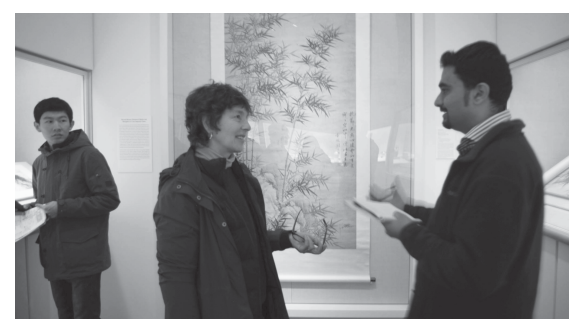

\section{- Interviews with Related Parties}

We interviewed an amateur Chinese painter in Singapore, a China-based freelance translator focused on art, a Chinese dealer in Chinese paintings, a New York-based professional working with Asian Art auctions, an assistant professor in Shanghai Normal University School of Art, and a Manager of Museum Technology. 